\title{
An Experimental Study of Stable Operating Conditions for a High Sensitivity Induction Gradiometer
}

\author{
Kunihisa Tashiro $^{1,2}$,Atsuya Kakiuchi. ${ }^{1}$, Ken-ich Moriizumi ${ }^{1}$ and Hiroyuki Wakiwaka ${ }^{1,2}$ \\ ${ }^{1}$ Department of Electrical and Electronic Engineering, Shinshu University \\ ${ }^{2}$ Spin Device Technology Center, Shinshu University
}

\begin{abstract}
Stable operating conditions for a high sensitivity induction gradiometer are studied experimentally. In order to reduce electrical interferences in our developed induction gradiometer, we construct a Faraday cage. It consists of copper mesh and wood, and is 2.0 $\mathrm{m} \times 1.8 \mathrm{~m} \times 1.8 \mathrm{~m}$ in size. Experimental results show that the inside of the cage can provide a quiet electrical environment where the electrical interference is suppressed. Choosing a suitable grounding point for the electronics, undesirable signals in the sensor output are cleaned. Observation of a weak magnetic field is also demonstrated.
\end{abstract}

Index Terms - Induction gradiometer, Faraday cage, electrical interferences, grounding

\section{INTRODUCTION}

An induction gradiometer (IG), based on definition of inductance, is a promising sensor which has the ability to detect weak magnetic fields from extremely low frequencies to those is the audible range $(0.01 \mathrm{~Hz} \sim 10 \mathrm{kHz})$. Although this kind of magnetic sensor was proposed in several papers [1-4], the technical details were usually not described. One of practical problems is how to provide stable operating conditions for the sensor. Misunderstanding SQUID sensors is a good example. Because SQUID sensors can detect weak magnetic fields, a magnetically shielded room is very important, but is not a must. This the sensor can operate outside of a magnetically shielded room, but electromagnetic interference and grounding problems have to be solved [5-6].

We have already reported an IG for a magnetocardiograph (MCG) [7]. The IG showed a few $\mathrm{mV}$ for a magnetic field of $100 \mathrm{pT}, 1 \mathrm{~Hz}$. The design of the pickup coil is based on a Brooks coil [8]. This shape of coil can achieve maximum inductance for a given length of winding wire, and the estimation error of the inductance is less than $3 \%$ [9-12]. Due to power line noise and electrical interference, however, the waveform of the target signal cannot be observed by an oscilloscope. This paper discusses stable operating conditions for an IG. Because the gains of the current-to-voltage converter (IV) and the instrumentation amplifier (IA) are larger than previous conditions, the electronics can not operate stably. First of all, we briefly describe the basis and specifications of our developed IG, and show electric and magnetic shielding performance of our constructed Faraday cage. One of the stable operating conditions is found through choosing a suitable grounding point for the IG's electronics. Observation of a weak magnetic field is also demonstrated.

Digital Object Identifier inserted by IEEE
II.IG

A schematic diagram of the IG is shown in Fig. 1. The pickup coil consists of two Brooks coils. The coil width is 30 $\mathrm{mm}$, the inner diameter is $60 \mathrm{~mm}$, the outer diameter is 120 $\mathrm{mm}$, and the distance between the two coils is $300 \mathrm{~mm}$. If there is an imbalance of magnetic flux density in the detection coil, it is transferred into electric current, and converted into output voltage. The flux-to-current transfer function and the cutoff frequency are defined by the coil. Although the cutoff frequency defined by the design of the pickup coil is $18 \mathrm{~Hz}$, IV with frequency compensation improves it as low as $0.2 \mathrm{~Hz}$. The output voltage is filtered by band elimination filter's (BEF's, $60 \mathrm{~Hz}$ and $180 \mathrm{~Hz}$ ) and a high pass filter (HPF, $0.1 \mathrm{~Hz}$ ), and is gained 110 times by IA. A low-noise DC power supply (E3630A Agilent) is used for the electronics. A photograph of our developed IG is shown in Fig. 2, and the specifications are summarized in Table 1.

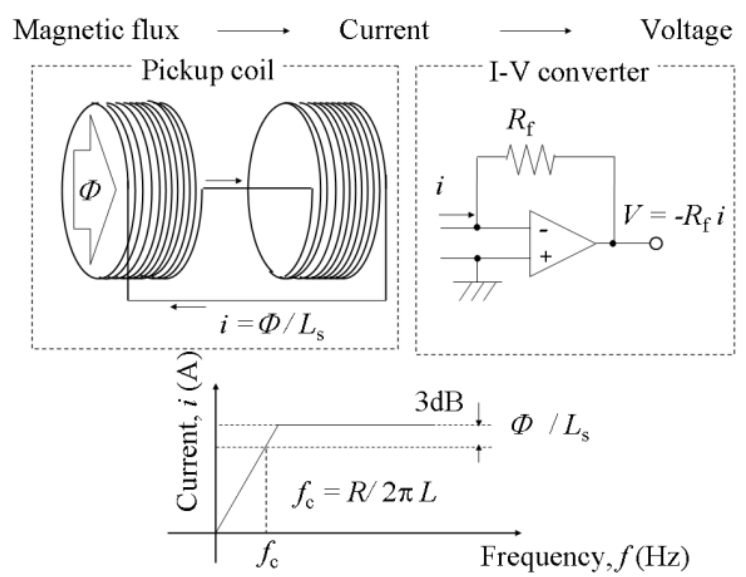

Fig. 1. Schematic diagram of an IG. The pickup coil has inductance $L[\mathrm{H}]$ and resistance $R[\Omega]$. When a flux linkage $\Phi[\mathrm{Wb}]$ crosses to one of the Brooks coils having inductance $L_{\mathrm{s}}[\mathrm{H}]$, a current $i[\mathrm{~A}]$ is induced. The current is transferred 
into electric current, and converted into output voltage. BEF's, HPF, and IA are not shown in this figure.

TABLE 1

SPECIFICATIONS OF THE IG

\begin{tabular}{lc}
\hline \hline Property & Value \\
\hline Diameter of pickup coil & $120 \mathrm{~mm}$ \\
Full length of pickup coil & $360 \mathrm{~mm}$ \\
Power supply for the electronics & $\pm 15 \mathrm{~V}$ \\
Cut off frequency $f_{\mathrm{c}}$ & $0.2 \mathrm{~Hz}$ \\
$\quad \begin{array}{l}\text { Flux to voltage transfer function, } T_{\mathrm{vb}} \\
\quad \text { (after the IV) } \\
\quad \text { (after the IA) }\end{array}$ & $15 \mathrm{mV} / \mathrm{nT}$ \\
\hline
\end{tabular}

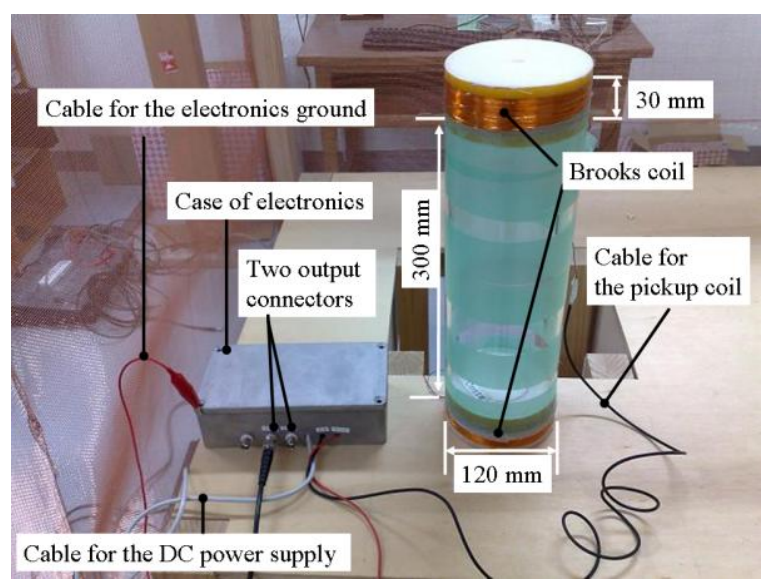

Fig. 2. A photograph of our developed IG. The electronics has two connectors for the output voltage of the IV and the IA. A shielding mesh is installed to the cable for the DC power supply. The cable for the pickup coil is a low-noise coaxial cable which has a semiconductor layer between outer conductor and inner insulator. Because of the low resistance of the pick-up coil, a usual twist pair cable permits a noise current to induce by moving the cable. The cable structure of the low-noise coaxial cable prevents the current noises less than $1 / 100$ [9]. The resistance of the cable for the electronics ground is less than 0.2 $\Omega$.

\section{FARADAY CAGE}

Our constructed Faraday cage consists of copper mesh and wood, and is $2.0 \mathrm{~m} \times 1.8 \mathrm{~m} \times 1.8 \mathrm{~m}$ in size. The copper mesh size is $2 \mathrm{~mm}$, and the diameter of the copper wire 0.45 $\mathrm{mm}$. No magnetic materials are used for avoiding magnetic noise.

We evaluated electric noise in our laboratory environment using a battery powered oscilloscope (TPS2014, Textronics) and a typical voltage probe. The measuring distance is $1 \mathrm{~m}$, and the height is $1 \mathrm{~m}$ from the floor. The measured value of voltage is divided by probe distance $(70 \mathrm{~mm})$, and converted into units of electrical field. The result is shown in Fig. 3. In our laboratory, a maximum measured value of $200 \mathrm{mV}_{\mathrm{p}-\mathrm{p}} / \mathrm{m}$ was observed in our environment. In contrast, the value was less than $20 \mathrm{mV}_{\mathrm{p}-\mathrm{p}} / \mathrm{m}$ inside of the cage.

In order to evaluate the magnetic shielding effect for low frequency fields of the cage, we measured magnetic noise using a fluxgate sensor (uMag-01, MEDA). Our defined measuring points are shown in Fig. 4. The fluxgate sensor is placed on the wooden base. The measured value of geomagnetic field is similar as $23 \mu \mathrm{T}$ at both positions. The measured amplitude of power line noise is also similar, as 10 to $100 \mathrm{nT}$, at the both positions. It means that the magnetic shielding effect of the cage was negligible. And the magnetic environment was similar at both positions. 


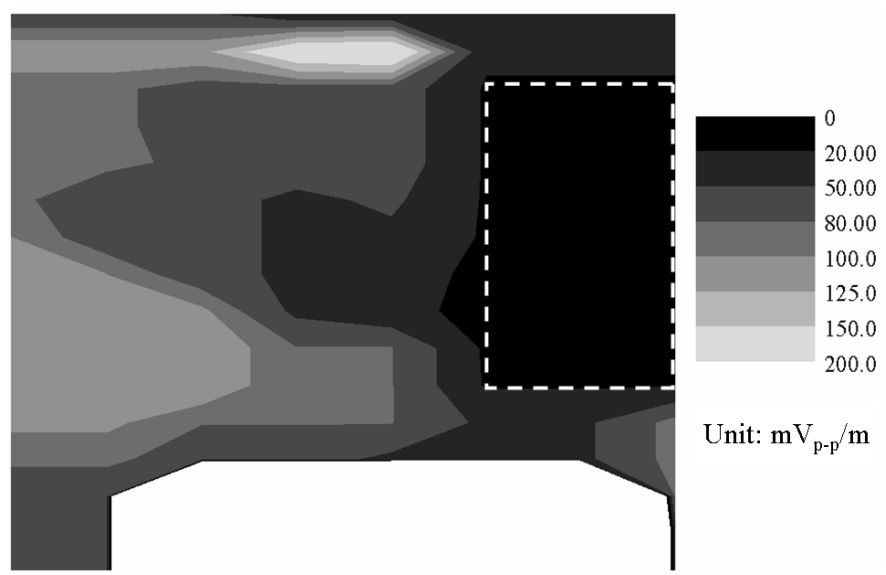

Fig. 3. Measured values of the electrical field in our laboratory. Dashed line area represents the position of the Faraday cage.

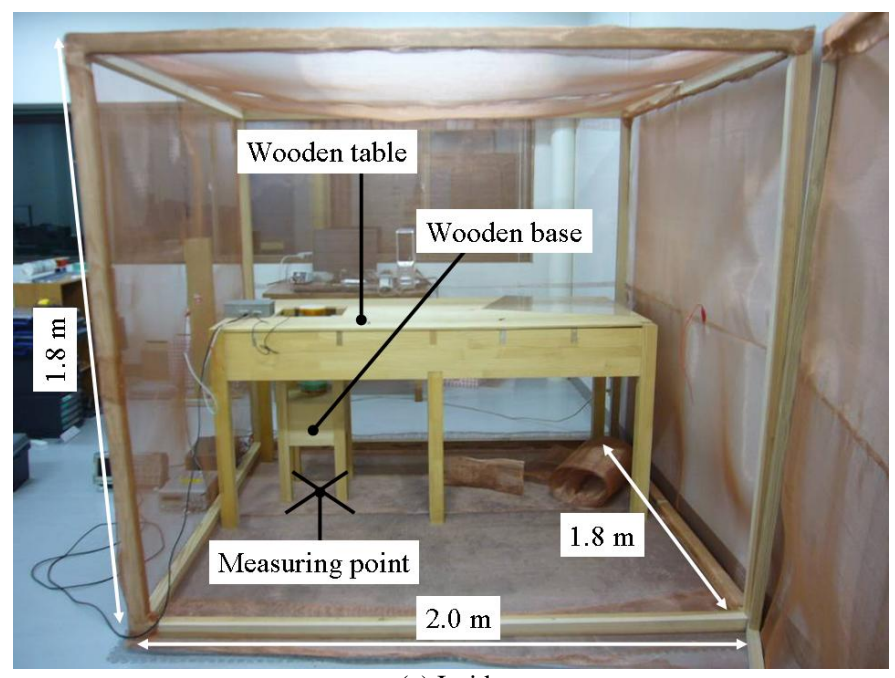

(a) Inside

\section{GROUNDING}

To find stable operating conditions for the IG, we choose four grounding points for the ground of the electronics (GE); (a) Faraday cage, (b) Case of electronics, (c) Floating ground, (d) Power line ground. The measuring points, defined in Fig. 4 , are also a parameter. We observed the IA's output voltage by the oscilloscope with a span of $2 \mathrm{sec}$. Observed range of the voltage is $5 \mathrm{~V} /$ div. Excluding the IG, all of the electrical equipments are placed the outside of the Faraday cage. A shielding mesh (SM) of a cable between DC power supply and the electronics is grounded to the Faraday cage. The measured values are summarized in Table 2 . If the value is described as 30 , it means that the IG does not work because the value is the limit level of IA's output voltage. As mentioned above, the magnetic environment was similar at both positions. However, the measured output signals of the IG are different. If the IG is placed the outside or the grounding point for the GE is the floating ground, the IG does not work. It is found that the Faraday cage can provide a

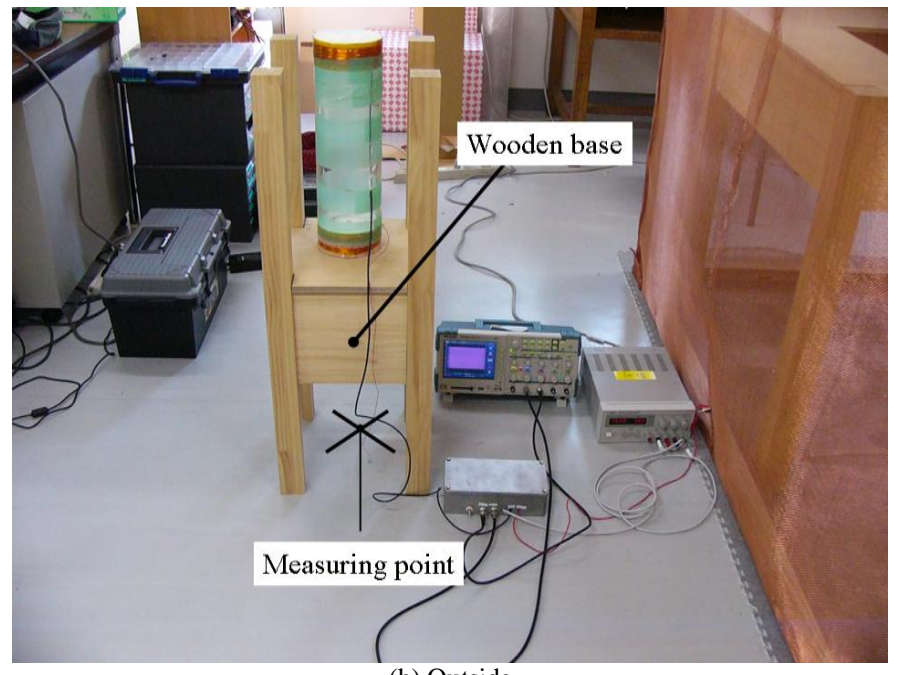

(b) Outside

Fig. 4 Definition of measuring points. Sensors are placed on the wooden base, and the sensing direction of the sensors is in the vertical direction. Excluding the IG, all of the electrical equipments are placed the outside of the Faraday cage.

quiet electrical environment for the the IG.

Although the cage can reject the electrical interference, wrong grounding point for the GE makes it worse. When the grounding point for the GE is the power line ground, the output voltage is lowest in Table 2. However, we conclude that it is not stable operating conditions. First reason is that the IV's output signal does not show appropriate offset voltage. An example of the pseudo stable signals is shown in Fig. 5 (a). The output voltage signal of the IV is also shown. Because the op-amp used in the IV has finite offset voltage, the output voltage of the IV should show a few volt of the offset value [8]. However, the IV's signal was close to zero. Second reason is that the other electrical interference to the IG is found as shown in Fig. 5(b). It seems that the IG detected quiet large magnetic field which corresponds to 100 nT. In our environment, this phenomenon was only found under this conditions and it occurred periodically in daytime. It may be caused by the conducting noise passing through ground line.

We also investigated the suitable grounding points for the 
SM and the case of the electronics (CE). If the SM was not grounded to the Faraday cage, the electrical interference became worse. Especially, if the grounding point for the GE is the case of the electronics, the IG does not work. From the results, we summarized the best conditions for the IG in Table 3.

TABLE 2

MEASURED VOLTAGE OF THE IA'S OUTPUT AS PARAMETERS OF MEASURING POSITION AND GROUNDING POINT FOR GE (UNIT: $\mathrm{V}_{\mathrm{P}-\mathrm{P}}$ )

\begin{tabular}{lcc}
\hline \hline & Inside & Outside \\
\hline (a) Faraday cage & 17.6 & $30^{*}$ \\
(b)Case of electronics & 18.0 & $30^{*}$ \\
(c) Floating ground & $30^{*}$ & $30^{*}$ \\
(d) Power line ground & 2.20 & $30^{*}$ \\
\hline \hline
\end{tabular}

* Saturation level of the IA's output voltage.
TABLE 3

BEST OPERATING CONDITIONS FOR THE IG

\begin{tabular}{lc}
\hline \hline Parameter & Condition \\
\hline Measuring space for the IG & Inside of the Faraday cage \\
Grounding point for & \\
GE: ground of the electronics & Faraday cage \\
SM: shielding mesh of the cable & Faraday cage \\
CE : case of the electronics & Faraday cage \\
\hline \hline
\end{tabular}

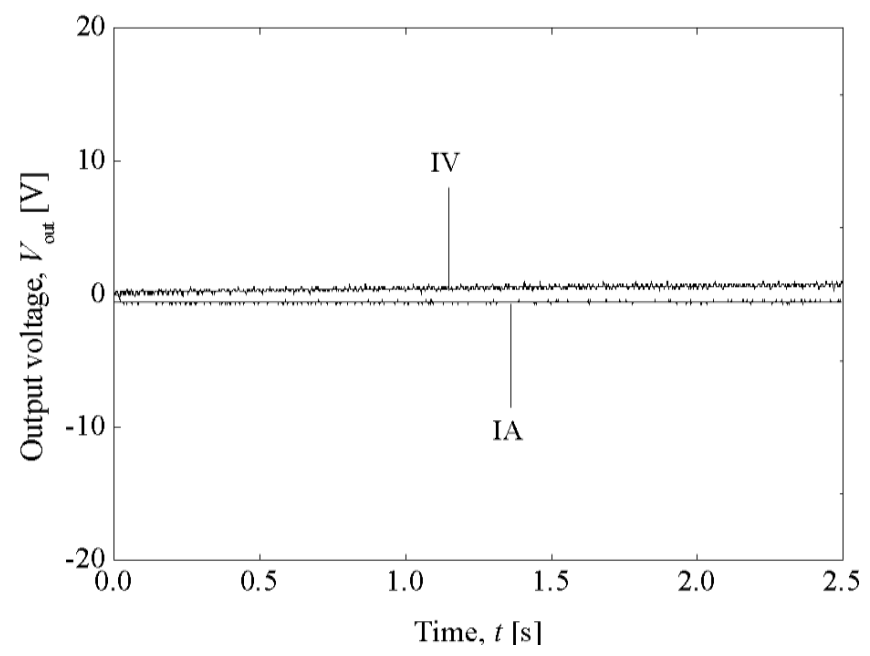

(a) Pseudo stable signals

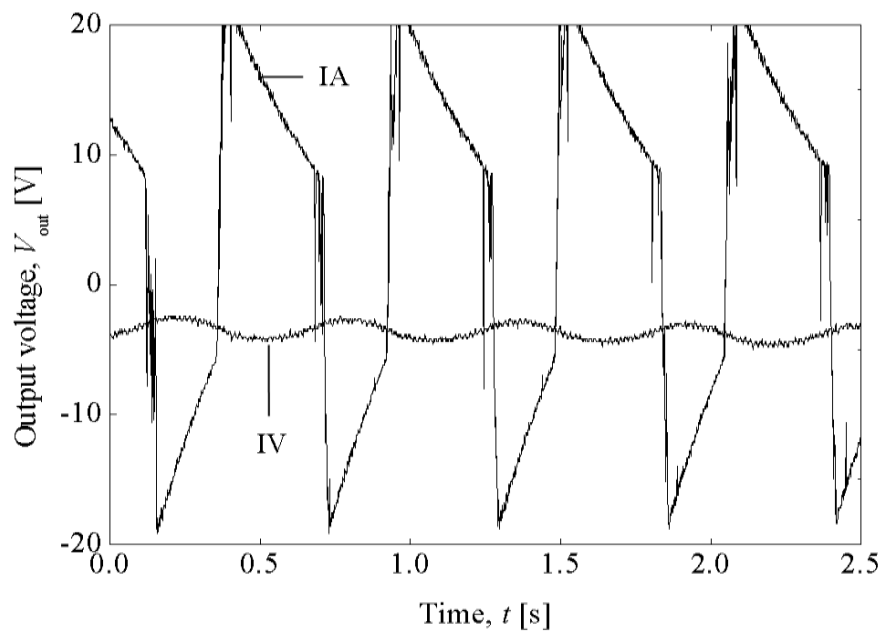

(b) Conducting noise signals

Fig. 5 Measured output voltage signal of the IG. IV represents the signal of the IV, and IA represents the output voltage of the IA.

\section{V.OBSERVATION OF A WEAK MAGNETIC FIELD}

We observed a weak magnetic field detected by the IG under the conditions summarized in Table 3. Because the pickup coil has a differential structure, we use a near field generated by a one turn coil having same diameter as the pickup coil. The distance between the detection coil and one turn coil is $10 \mathrm{~mm}$, and a small input current, $0.1 \mathrm{~mA}_{\mathrm{p}-\mathrm{p}}$ at 1 $\mathrm{Hz}$, is fed to the one turn coil. The measured output voltage signal of the IA observed by the oscilloscope is shown in Fig. 
6(a). Because a battery powered oscilloscope is free from the power line ground problem, it is one of the good instruments. Although computer based instruments have a lot of advantage of signal analysis, it has possibility to be a source of electrical interference problems. We also observed the signal by a LabView system (NI Corp.). The hardware is an A/D converter with 16 bit resolution (NI9215) which is installed to the CompactDAQ, and the software is LabView Full Development System (Ver. 8.2). The acquired signal is filtered by a digital LPF of $30 \mathrm{~Hz}$. The signal is shown in Fig. 6(b). In both case, the field was clearly detected by the IG. In contrast, if the grounding point for GE was the power line ground, the IG did not detect the signal.

\section{CONCLUSION}

We have constructed a Faraday cage, and studied stable operating conditions for our developed induction gradiometer experimentally. Although the magnetic shielding effect of the cage was negligible, it provided a quiet electrical environment and a suitable grounding point for the electronics. Because the amplitude of the geomagnetic field inside the cage is as same as outside the cage, the small vibrations of the pickup coil become harmful disturbances for the measurement, which also appears in the output signal. We will try to solve this problem in the near future.

\section{REFERENCE}

[1] S. A. Macintyre, "A Portable Low-Noise Low-Frequency 3-Axis Search Coil Magnetometer," IEEE Trans. on Magn., vol. 16, pp. 761-763, 1980.

[2] K. P. Estola and J. Malmivuo, "Air-Core Induction-Coil Magnetometer Design," Journal of Physics E-Scientific Instruments, vol. 15, pp. 1110$1113,1982$.

[3] R. J. Prance, T. D. Clark, and H. Prance, "Compact room-temperature induction magnetometer with superconducting quantum interference device level field sensitivity," Rev. Sci. Instrum., vol. 74, pp. 3735-3739, Aug. 2003.

[4] S. Tumanski, "Induction coil sensors - a review," Measurement Science \& Technology, vol. 18, pp. R31-R46, Mar 2007.

[5] N. Ishikawa, K. Nagata, H. Saito, N. Kasai, and S. Kiryu, "Effect of RF interference on characteristics of dc SQUID system," IEEE Trans. Appl. Super., vol. 3, pp. 910-1913, 1993.

[6] K. Tashiro, I. Sasada, K. Yoshitomi, and K. Kazami , "Reduction of RF noise for Stable SQUID Sensor Operation inside a Cylindrical Magnetic Shield," JSAEM Studies in Appl. Electromag. and Mech., vol. 14, pp. 249-256, 2003.

[7] F. W. Grover, Inductance Calculations, Dover Phenix Editions, 2004.

[8] A.Kakiuchi, A. Matsuoka, K. Tashiro, and H. Wakiwaka, "Development of induction gradiometer for magnetocardiography", Proc. of SEAD20, pp. 519-522, 2008. in Japanese.

[9] K. Tashiro, "Optimal Design of an Air-Core Induction Magnetometer for Detecting Low-Frequency Fields of Less than 1 pT," J. Magn. Soc. Jpn., vol. 30, pp. 439-442, 2006.

[10] K. Tashiro, "Proposal of coil structure for air-core induction magnetometer," Proc. IEEE Sensor 2006, pp. 939- 942, 2006.

[11] K. Tashiro, "Broadband air-core Brooks-coil induction magnetometer," SICE - ICCAS 2006, vol. TA07-2, p. 4 Pages, 2006.

[12] K. Tashiro, H. Wakiwaka, A. Kakiuchi, and A. Matsuoka, "Comparative study of air-core coil design for induction magnetometer with current-tovoltage converter", Proc. of second international conference on sensing technology (ICST2007), pp. 590-594, 2007.

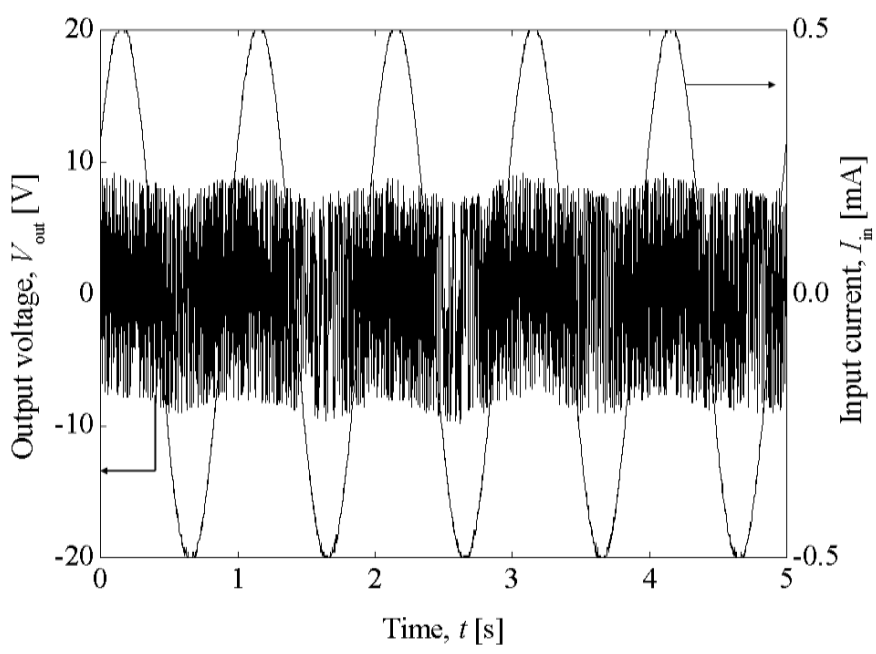

(a) Measured by the oscilloscope

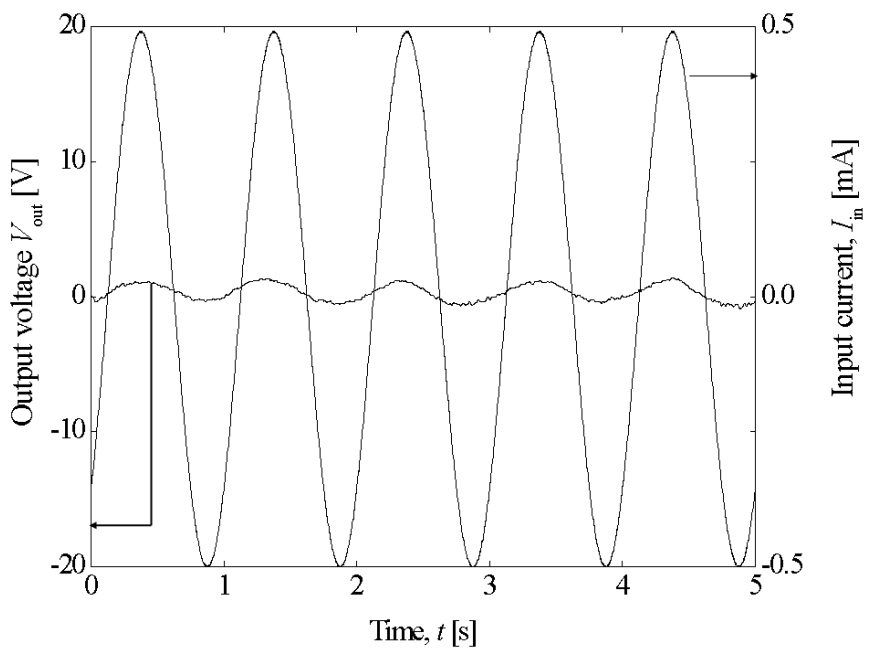

(b) Measured by the LabView system with a digital LPF of $30 \mathrm{~Hz}$

Fig. 6 A demonstration of observing a weak magnetic field detecting by the IG under the conditions described in Table 3. The output voltage represents the IA's signal, and the input current represents the current signal which is fed to the one turn coil. The distance between the detection coil and the one turn coil is $10 \mathrm{~mm}$.

\section{ACKNOWLEDGMENT}

This work was supported in part by the Japanese Ministry of Education, Science, Sports and Culture, Grant-in-Aid for Young Scientists (B), under Grant 19760048, 2007-2008. 